\title{
Localisation, chiral symmetry and confinement in QCD and related theories
}

\author{
Matteo Giordano* \\ ELTE Eötvös Loránd University, Institute for Theoretical Physics, Pázmány P. s. 1/A, H-1117, \\ Budapest, Hungary, and \\ MTA-ELTE Lendület Lattice Gauge Theory Research Group, Pázmány P. s. 1/A, H-1117, \\ Budapest, Hungary \\ E-mail: giordano@bodri.elte.hu
}

I discuss recent results on the relation between the localisation of low-lying Dirac eigenmodes, the restoration of chiral symmetry, and deconfinement in QCD and QCD-like models, providing evidence of a close connection between the three phenomena.

XIII Quark Confinement and the Hadron Spectrum - Confinement2018

31 July - 6 August 2018

Maynooth University, Ireland

${ }^{*}$ Speaker. 


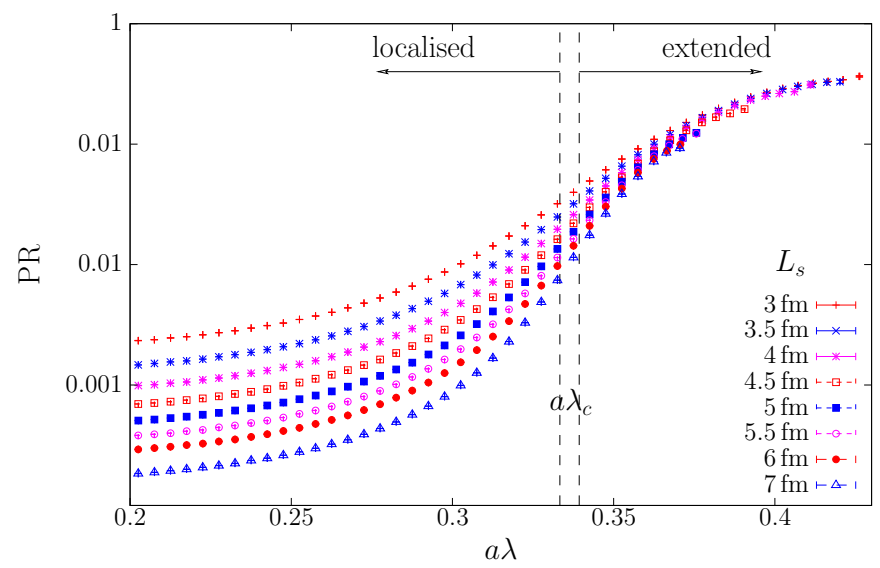

Figure 1: Participation ratio of the low Dirac modes in high-temperature QCD (here $T \simeq 400 \mathrm{MeV}$ ), obtained on the lattice with staggered fermions. Data from Ref. [11].

\section{Introduction}

It is well known that QCD at finite temperature undergoes a deconfining and chirally-restoring transition, or, more precisely, that QCD displays an analytic crossover in the range $T \simeq 145-165$ $\mathrm{MeV}$ in which both confining and chiral properties change radically. The pseudocritical temperatures for the chiral and deconfining transitions, defined from the position of the peaks of the chiral susceptibility and of the quark entropy, respectively, are equal within errors [1], 国]. For the sake of definiteness we will take $T_{c} \simeq 155 \mathrm{MeV}$. The close relation between these two very different phenomena is not unique to QCD, but is found also in other QCD-like theories, like, e.g., SU(3) pure gauge theory in 3+1 [3] and 2+1 dimensions [ [4], and $N_{f}=3$ QCD with unimproved rooted staggered fermions on $N_{T}=4$ lattices [⿰氵⿹丁口⿹丁口 . The nature of this relation is however not fully understood yet.

It is also well known that chiral symmetry breaking originates from the accumulation of eigenvalues of the Dirac operator near the origin [6]: the spectral density is finite at the origin below $T_{c}$, while it vanishes above it. The question is then if such an accumulation, or the lack thereof, is related to the confining properties of the theory. A possible link, or at least a tool to study the relation between deconfinement and chiral symmetry restoration, is a third phenomenon that also takes place at $T_{c}$, namely the localisation of the lowest Dirac modes.

It is by now well established that while below $T_{c}$ all the Dirac modes are extended throughout the whole system, above $T_{c}$ the low modes are localised, up to a temperature-dependent critical point $\lambda_{c}(T)$ in the spectrum where a localisation/delocalisation transition, or Anderson transition, takes place [7, 8, 9, 10, 11, 12, 13]. The simplest way to observe localisation is to measure the participation ratio (PR) of the eigenmodes, which measures the fraction of spacetime effectively occupied by a mode. For normalised eigenvectors $\psi(x)$ of the Dirac operator, computed on a hypercubic lattice of spatial volume $V=N_{s}^{3}$ and temporal size $N_{T}$ (in lattice units), one has

$$
\mathrm{PR} \equiv \frac{1}{V N_{T}}\left[\sum_{x}\left|\psi^{\dagger}(x) \psi(x)\right|^{2}\right]^{-1},
$$

where $\psi^{\dagger}(x) \psi(x)$ denotes the scalar product in colour and Dirac space. As the volume of the 
system is increased, the PR remains constant for an extended mode, while it goes to zero for a mode localised in a finite region. Localisation of the low modes in high-temperature QCD can be seen in Fig. 1.

In this contribution, after a brief discussion of the main tools required in the study of localisation, I will review what is known about localisation in QCD. I will then discuss a possible mechanism for localisation, and how this mechanism relates to the confining and chiral properties of QCD. I will then proceed to discuss localisation in QCD-like theories in 3+1 dimensions, briefly discuss the possible role of topology, and show some preliminary results for QCD-like theories in $2+1$ dimensions.

\section{Numerical studies of localisation}

The main tools required to study localisation in QCD are the theory of disordered Hamiltonians, Lattice Gauge Theory, and Random Matrix Theory, which I now briefly review.

\subsection{Disordered Hamiltonians}

Disordered Hamiltonians aim at describing systems containing some form of disorder. The most famous example is the Hamiltonian of the Anderson model (AM) for electrons in "dirty" conductors [14], which consists of the usual tight-binding Hamiltonian supplemented with a random on-site potential $\varepsilon_{\vec{x}}$, mimicking the presence of impurities in the crystal,

$$
H_{\vec{x}, \vec{y}}^{\mathrm{AM}}=\varepsilon_{\vec{x}} \delta_{\vec{x}, \vec{y}}+\sum_{\mu=1}^{3}\left(\delta_{\vec{x}+\hat{\mu}, \vec{y}}+\delta_{\vec{x}-\hat{\mu}, \vec{y}}\right) .
$$

In the simplest version of the model, the $\varepsilon_{\vec{x}}$ are drawn from a uniform distribution on the interval $\left[-\frac{W}{2},+\frac{W}{2}\right]$, whose width $W$ measures the amount of disorder in the system. While in the absence of disorder $(W=0)$ the electron states are delocalised Bloch waves, as soon as some disorder is put into the system $(W \neq 0)$ localised modes appear at the band edge, beyond a critical energy $E_{c}(W)$, called "mobility edge". As the amount of disorder is increased, $E_{c}(W)$ moves towards the band center, until a critical disorder $W_{c}$ is reached: for $W>W_{c}$ all modes are localised, and the metal becomes an insulator. As one crosses the mobility edge the system undergoes a second-order phase transition with divergent correlation length $\xi(E) \simeq\left|E-E_{c}\right|^{-v}$, known as Anderson transition. The analogue of $E_{c}(W)$ in QCD is $\lambda_{c}(T)$, with temperature playing the role of control parameter of the amount of disorder in the system. By contrast with the AM, in QCD all the modes become extended below $T_{c}$.

The AM discussed here is the simplest one, with diagonal disorder only, but other versions exist. One that is relevant to us is the so-called unitary Anderson model (UAM), which includes also off-diagonal disorder in the form of random phases in the hopping terms, mimicking the presence of a random magnetic field,

$$
H_{\vec{x}, \vec{y}}^{\mathrm{UAM}}=\varepsilon_{\vec{x}} \delta_{\vec{x}, \vec{y}}+\sum_{\mu=1}^{3}\left(\delta_{\vec{x}+\hat{\mu}, \vec{y}}+\delta_{\vec{x}-\hat{\mu}, \vec{y}}\right) e^{i \phi_{\vec{x}, \vec{y}}}, \quad \phi_{\vec{y}, \vec{x}}=-\phi_{\vec{x}, \vec{y}} .
$$

Here "unitary" refers to the symmetry class in the sense of Random Matrix Theory, discussed below. The AM of Eq. 2.1) belongs instead to the orthogonal class. The change in symmetry in turn affects the critical behaviour at the Anderson transition. 


\subsection{Lattice Gauge Theory}

Most nonperturbative studies of QCD are based on Lattice Gauge Theory (LGT), which deals with the gauge theory functional integral in Euclidean space discretised on a finite lattice, which is typically a hypercube of spacing $a$ of sizes $N_{S}$ and $N_{T}$ in the spatial and temporal directions, respectively. Fermion fields $\psi(n), \bar{\psi}(n)$ live on the lattice sites $n$, while gauge fields $A_{\mu}(x)$ are replaced by parallel trasporters, $U_{\mu}(n)=\operatorname{Pexp}\left\{i g \int_{a n}^{a n+a \hat{\mu}} A_{\alpha}\left(x^{\prime}\right) d x^{\prime \alpha}\right\}$ living on the lattice edges $(n, n+\hat{\mu})$. Periodic boundary conditions are usually imposed on the fields in the spatial directions. At finite temperature the lattice size $1 / T=a N_{T}$ in the temporal direction is kept fixed in physical units, and appropriate boundary conditions are imposed on the fields (periodic for bosons and antiperiodic for fermions). A detailed review of LGT is way beyond the scope of this paper. There are however two points worth emphasising. The first important point is that in LGT the gauge theory functional integral turns in practice into the partition function of a statistical system,

$$
Z=\int \mathscr{D} U \int \mathscr{D} \psi \int \mathscr{D} \bar{\psi} e^{-S_{\text {gauge }}[U]-\bar{\psi}\left(D_{\mathrm{lat}}[U]+m\right) \psi},
$$

where the integration extends over a large but finite number of degrees of freedom. The infinitevolume limit $V \rightarrow \infty$ and the continuum limit $a \rightarrow 0$ are eventually taken. Here $S_{\text {gauge }}[U]$ and $D_{\text {lat }}[U]$ are respectively a discretised version of the Yang-Mills action and of the Dirac operator. The case I will be focussing on is that of the staggered discretisation,

$$
\left[D_{\text {stag }}\right]_{n, n^{\prime}}=\frac{1}{2} \sum_{\mu} \eta_{\mu}(n)\left[U_{\mu}(n) \delta_{n+\hat{\mu} . n^{\prime}}-U_{\mu}(n-\hat{\mu})^{\dagger} \delta_{n-\hat{\mu} . n^{\prime}}\right], \quad \eta_{\mu}(n)=(-1)^{\sum_{\alpha<\mu} n_{\alpha}} .
$$

The second relevant point here is that the staggered operator is just $i$ times the Hamiltonian of a quantum mechanical system with purely off-diagonal disorder, provided by the gauge links $U_{\mu}(n)$, and so the Dirac operator can be studied just like a disordered Hamiltonian.

\subsection{Random Matrix Theory}

Random Matrix Theory (RMT), quite unsurprisingly, studies matrices with random entries, examples of which are the AM Hamiltonian and the lattice Dirac operator. RMT however typically deals with dense matrices, for which certain statistical properties of the spectrum are universal, depending only on the symmetry class of the matrix ensemble. An important universal property of dense RMT ensembles is the distribution of the unfolded level spacings, i.e., the distance between consecutive eigenvalues divided by the average level spacing in the relevant spectral region. The unfolded spectrum is defined by mapping the eigenvalues $\lambda_{i}$ to $\lambda_{i} \rightarrow x_{i}=\int{ }^{\lambda_{i}} d \lambda \rho(\lambda)$, where $\rho(\lambda) \equiv\left\langle\sum_{n} \delta\left(\lambda-\lambda_{n}\right)\right\rangle$ is the spectral density, and $\langle\ldots\rangle$ denotes ensemble averaging. After unfolding, the spectral density becomes identically 1 throughout the spectrum. The unfolded level spacing distribution (ULSD) $P(s)$ is the probability distribution of $s_{i}=x_{i+1}-x_{i}$ over the matrix ensemble. This quantity can be computed analytically for the so-called Gaussian ensembles, and by universality it describes the unfolded spectrum for all models in a given symmetry class. The ULSD is very well approximated by the Wigner's surmise, $P(s)=a s^{b} e^{-c s^{2}}$, with class-dependent constants $a, b, c$. By contrast, for independently fluctuating eigenvalues (Poisson statistics) one finds $P(s)=e^{-s}$. 

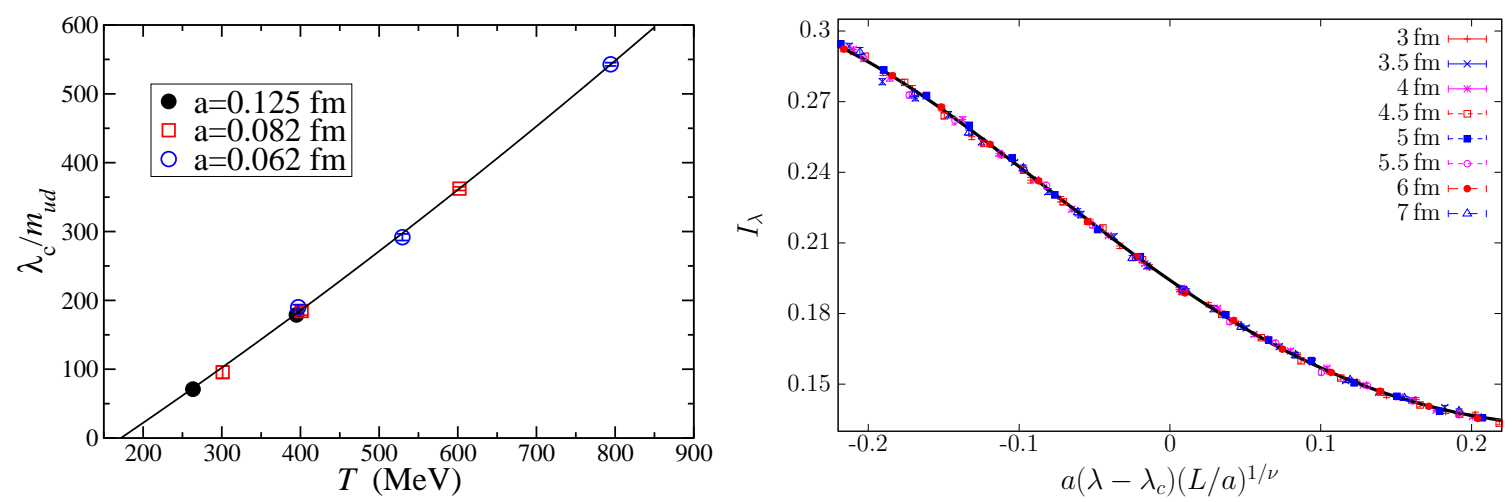

Figure 2: (Left.) Dependence of the mobility edge on temperature in QCD. Figure from Ref. [10]. (Right.) Scaling function for $I_{\lambda}$ at the Anderson transition in QCD (see text for details). Data from Ref. [11].

For the sparse matrices we are interested in, it turns out that the spectral statistics depend on the spectral region, and are connected to the localisation properties of the eigenmodes: extended modes obey the appropriate type of RMT statistics given the symmetries of the model, while localised modes obey Poisson statistics, fluctuating independently. This connection can be used to determine precisely the position of the mobility edge $\lambda_{c}$ by means of a finite-size-scaling study of spectral statistics [15]. Indeed, given a dimensionless quantity $\mathscr{O}(\lambda, L)$ derived from the ULSD and computed locally in the spectrum, i.e., in an infinitesimal neighbourhood of $\lambda$, one expects under the usual scaling hypothesis that in the vicinity of $\lambda_{c}$ it depends on the system size $L$ as $\mathscr{O}(\lambda, L)=O(\xi(\lambda) / L)=O\left(\left|\lambda-\lambda_{c}\right|^{-v} / L\right)$. Analyticity in a finite volume then imposes $\mathscr{O}(\lambda, L)=f\left(\left(\lambda-\lambda_{c}\right) L^{1 / v}\right)$. This relation can be used to determine $\lambda_{c}$ and the critical exponent $v$, as well as the critical value $\mathscr{O}_{c}=\mathscr{O}\left(\lambda_{c}, L\right)$, which is volume-independent, and expected to be determined by a universal critical statistics. A convenient observable turns out to be the integrated ULSD,

$$
I_{\lambda}=\int_{0}^{s_{0}} d s P(s)
$$

where $s_{0}$ is the crossing point of the appropriate Wigner's surmise and the exponential function.

\section{Localisation in Lattice QCD}

As already anticipated in the Introduction, while all the Dirac modes are delocalised in the low-temperature phase of QCD, above $T_{c}$ the low modes are localised for $\lambda<\lambda_{c}(T)$, with a secondorder phase transition (Anderson transition) at $\lambda_{c}(T)$. Localised modes have been observed with different fermion discretisations (staggered [7, 10], overlap [8, 13], domain wall [12]). Most quantitative studies have been carried out with staggered fermions, on which I will mostly focus here.

\subsection{Numerical results}

The dependence of the mobility edge on temperature was studied in Ref. [10], using 2+1 flavours of 2-stout improved rooted staggered fermions with physical quark masses [1]. As can be seen in Fig. 目 (left), $\lambda_{c}(T)$ extrapolates to zero at a temperature compatible with $T_{c}$. Since it is a feature of the spectrum, the mobility edge is expected to renormalise like a quark mass [16, 10], 
and so $\frac{\lambda_{c}}{m_{u d}}$ with $m_{u d}$ the light quark mass is expected to be the physically relevant, renormalisationgroup-invariant quantity. This ratio seems indeed to be independent of the lattice spacing, so that localisation is expected to survive the continuum limit.

Critical properties at the mobility edge were studied in Ref. [11] with the same type of fermions on $N_{T}=4$ lattices at $\beta=3.75$, corresponding to a lattice spacing of $a=0.125 \mathrm{fm}$ and a temperature of $T=394 \mathrm{MeV}=2.6 T_{c}$. By means of a finite-size-scaling analysis [see Fig. 2 (right)] the critical exponent $v$ was found to be $v_{\mathrm{QCD}}=1.46(3)$, in agreement with that obtained for the 3D UAM [17]. Further studies showed that also the multifractal exponents characterising the eigenvectors at criticality match those of the 3D UAM [18].

\subsection{Polyakov lines, the "sea/islands" picture and the Dirac-Anderson Hamiltonian}

It is a bit surprising that QCD and UAM share the same critical properties at the Anderson transition. While the unitary symmetry class is indeed the appropriate one for staggered fermions, and the dimensionality of high-temperature QCD is indeed expected to be reduced from 4 to 3 , at first sight it is hard to spot any source of 3D diagonal disorder. The staggered Dirac operator has in fact only off-diagonal 4D disorder, for which different critical properties are expected.

The source of diagonal disorder was first identified by the authors of Ref. [19] in the spatial fluctuations of the Polyakov lines. For a perfectly ordered configuration with Polyakov lines aligned along the identity and trivial spatial links, the spectrum displays a sharp gap corresponding to the lowest Matsubara frequency. For realistic configurations the ordering is partial, with a "sea" of ordered Polyakov lines and "islands" of fluctuations away from the identity, so the gap turns into an effective gap, below which the spectral density is small. Living on the "islands" is "energetically" favourable, allowing to reduce the eigenvalue and penetrate the gap, the price to pay being that the mode has to be localised in these "traps". The argument was later extended from SU(2) to SU(3) in Ref. [20], and has been tested in several ways: on SU(2) configurations [19], in a toy model for QCD [20], and most recently in QCD with domain-wall [12] and overlap fermions [13].

A shortcoming of this argument is that it does not explain why there is no localisation below $T_{c}$. The absence of islands cannot be the answer, since there is no such a thing as islands in the AM, which displays localised eigenmodes nonetheless. This is simply due to the presence of fluctuations in the disorder, which certainly are present in the Polyakov lines also in the low-temperature phase. In fact, what one has below $T_{c}$ is an effectively $4 \mathrm{D}$ system with (relatively weak) off-diagonal disorder, as one would expect by taking the Dirac operator at face value. For this system, no localised modes at the band center are expected.

A step in understanding this issue is made by showing that the staggered operator is formally equivalent to a set of coupled 3D AMs [21, 22, This is done by separating the temporal hoppings, treated as an unperturbed, "free" Hamiltonian, from the spatial hoppings, treated as the interaction, and then diagonalising the free Hamiltonian. After diagonalisation of its free part, the "DiracAnderson Hamiltonian" $H=-i D_{\text {stag }}$ reads

$$
\begin{gathered}
H_{\vec{x}, \vec{y}}=\delta_{\vec{x}, \vec{y}} D(\vec{x})+\sum_{j=1}^{3} \frac{\eta_{j}(\vec{x})}{2 i}\left[\delta_{\vec{x}+\hat{\jmath}, \vec{y}} V_{+j}(\vec{x})-\delta_{\vec{x}-\hat{\jmath}, \vec{y}} V_{-j}(\vec{x})\right] \\
{[D(\vec{x})]_{a k, b l}=\eta_{4}(\vec{x}) \sin \omega_{a k}(\vec{x}) \delta_{a b} \delta_{k l}, \quad\left[V_{ \pm j}(\vec{x})\right]_{a k, b l}=\frac{1}{N_{T}} \sum_{t=0}^{N_{T}-1} e^{i \frac{2 \pi t}{N_{T}}(l-k)}\left[\tilde{U}_{ \pm j}(t, \vec{x})\right]_{a b} .}
\end{gathered}
$$


Here $\tilde{U}_{ \pm j}$ are the spatial links in the "uniform temporal diagonal gauge", or Polyakov gauge, defined by the condition

$$
U_{4}(t, \vec{x})=[P(\vec{x})]^{\frac{1}{N_{T}}}=\operatorname{diag}\left(e^{i \frac{\phi_{1}(\vec{x})}{N_{T}}}, \ldots, e^{i \frac{\phi_{N_{C}}(\vec{x})}{N_{T}}}\right)
$$

where $e^{i \phi_{a}(\vec{x})}, a=1, \ldots, N_{c}$, are the eigenvalues of the Polyakov line at $\vec{x}$, subject to the condition $\sum_{a} \phi_{a}(\vec{x})=0$. The unperturbed eigenvalues are determined by the effective Matsubara frequencies,

$$
\omega_{a k}(\vec{x})=\frac{1}{N_{T}}\left(\pi+\phi_{a}(\vec{x})+2 \pi k\right),
$$

where the indices correspond to the spatial site $\vec{x}$, colour $a$, and temporal momentum $k=0, \ldots, N_{T}-$ 1. One then finds precisely $N_{T} 3 \mathrm{D}$ AMs with diagonal disorder provided by the Polyakov line phases through the effective Matsubara frequencies, plus off-diagonal disorder coming from the interaction. These models are also coupled by the interaction, and the strength of the coupling is related to the correlation between time slices: the stronger the correlation, the weaker the coupling.

Unlike the simple AMs discussed previously, the strength of the disorder (both diagonal and off-diagonal) is bounded here (the matrices $V_{ \pm j}(\vec{x})$ can be shown to be unitary), so one cannot simply induce localisation by increasing the noise. However, in the two phases of QCD the nature of the disorder is different. In the disordered phase there are weak correlations among time slices, and no structure in the diagonal noise, so strong coupling of the AMs and uncorrelated diagonal noise. In the ordered phase there are strong correlations among time slices due to ordering of the Polyakov lines, which also provide a "sea" in which "islands" of convenient localising centers are found. This implies weak coupling of the AMs and correlated diagonal disorder. Studies in a QCDbased toy model [21] show that both the correlations of time slices and the presence of islands are needed to localise the low modes.

\subsection{Chiral symmetry restoration and localisation from deconfinement?}

It is now time to ask the important question: how are deconfinement, chiral symmetry restoration, and localisation of the low modes related? Although I cannot give a definite answer, I can at least list a few points that the answer should address.

1. Deconfinement changes the effective dimensionality of the Dirac-Anderson system and creates an effective gap in the spectrum.

At low temperature the AMs are strongly coupled, so the temporal momentum is effectively one more dimension, and the Dirac-Anderson system is then effectively 4D. This is not surprising, but then there is nothing to gain in recasting the Dirac operator in the Dirac-Anderson form, and the purely off-diagonal nature of the disorder is already captured in the usual form of the staggered operator. At high temperature instead the coupling is weak, and the system acts as a collection of 3D AMs with diagonal disorder, weakly interacting with each other.

2. Localisation requires the presence of an effective gap and decoupling of the AMs.

In the presence of an effective gap, islands are convenient for localisation, but if the AMs are too strongly coupled then the mixing of modes can prevent localisation. This was understood studying the toy model of Ref. [21]. 

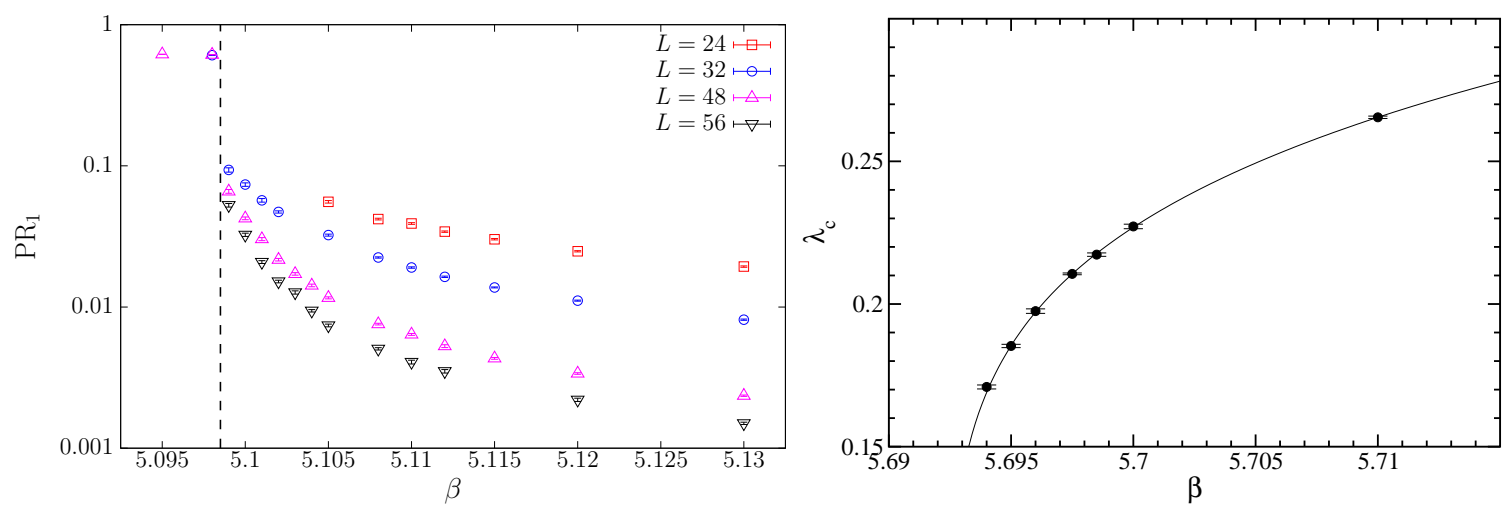

Figure 3: (Left.) PR of the lowest mode as a function of the coupling for $N_{f}=3$ unimproved staggered fermions on $N_{T}=4$ lattices. Data from Ref. [23]. (Right.) Mobility edge as a function of the coupling in $\mathrm{SU}(3)$ pure gauge theory. Figure from Ref. [24].

3. Chiral symmetry breaking, i.e., a finite density of near-zero modes, requires that the AMs be coupled, and that a finite density of small unperturbed modes be present.

Also this was understood studying the toy model of Ref. [21]: eigenmodes do not accumulate near the origin if there is no mixing of temporal momenta, and if there are not enough low unperturbed modes.

The conclusion is that deconfinement seems to precisely provide the conditions for the localisation of Dirac modes, and to remove the conditions for chiral symmetry breaking. If the picture above is correct, this should happen in gauge theories on quite general grounds, since nothing specific to QCD has been used: all that is required is the ordering of the Polyakov lines. If so, then the coincidence of deconfinement, chiral symmetry restoration and localisation of the low modes should be a rather general phenomenon. This is what I am going to discuss next.

\section{Localisation in 3+1D QCD-like theories}

In this section I review results about the connection between deconfinement, chiral symmetry restoration and localisation in 3+1D QCD-like theories. To study this connection, theories with a genuine phase transition are better suited, since they provide a clean-cut situation with clearly separated phases.

The first case I will discuss is $N_{f}=3$ QCD with unimproved rooted staggered quarks on lattices with $N_{T}=4$, studied in Ref. [23]. For light enough quarks this model displays a first order phase transition at some critical value of the coupling [[]], with both the Polyakov loop expectation value and the chiral condensate showing a jump. Although this transition is a lattice artefact that does not survive the continuum limit, nevertheless this model considered at fixed lattice spacing is a perfectly good statistical model where we can test our ideas about deconfinement, chiral restoration and localisation. In Fig. 3 (left) I show the participation ratio of the lowest mode as a function of the lattice gauge coupling $\beta$ for several volumes. While below the critical coupling $\beta_{c}$ the PR does not change much with the volume, above $\beta_{c}$ it keeps decreasing as the system size is increased, meaning that the lowest mode is delocalised in the low-temperature phase and localised in the high-temperature phase. 
Another interesting model is the SU(3) pure gauge theory, which has a first order deconfining transition, in correspondence of which the valence quark condensate shows a transition as well [3]. The authors of Ref. 24 have determined the mobility edge as a function of the gauge coupling, and they have found that it vanishes at a value compatible with the critical $\beta$ [see Fig. B (right)]. Again, localised modes appear at deconfinement.

It is worth mentioning that the same behaviour was found in Ref. [22] in the toy model for QCD of Ref. [21]. Localised modes were found also in the high-temperature phase in SU(2) pure gauge theory [9], although a detailed study of the mobility edge as a function of temperature was not performed.

\section{Topology}

In this section I briefly discuss the possible role played by topology in the localisation of low modes. The authors of Ref. [7] suggested that localised modes are related to the localised zero modes associated to instantons at finite temperature. However, it was found in Ref. [24] that even in the $\mathrm{SU}(3)$ pure gauge theory case, where the instanton density is higher than in QCD, they contribute for no more than $60 \%$ at $T_{c}$, and this fraction vanishes rapidly as $T$ increases.

The authors of Ref. [12] made a different proposal. They showed that the localised low modes prefer locations with larger action density and topological charge density, while the delocalised higher modes show no particular preference. Moreover, they suggested that the localisation might take place in correspondence with $L$ and $\bar{L}$-type monopole-instanton pairs. On the one hand, this would perfectly match the best "islands" identified by the Polyakov-line argument, which the localised modes indeed seem to prefer. On the other hand, these topological objects could be related to confinement, as opposed to instantons. In any case, the role of topology is far from being understood, and more studies are certainly required.

\section{Localisation in QCD-like theories in 2+1D}

In this section I discuss QCD-like theories in 2+1 dimensions. Based on our general expectations about the relation between confining properties and the Dirac spectrum, dimensionality should play no particular role, as long as it is possible to have a deconfined phase of the theory, and as long as a localisation/delocalisation transition is possible. These theories should therefore show a close connection between deconfinement, chiral transition and localisation of the low modes.

However, quite a few things are different from the $3+1 \mathrm{D}$ case, most notably the absence of chiral symmetry in three dimensions. Of course one could simply check for a nonzero spectral density at the origin without bothering about symmetries, but, as a matter of fact, for an even number $N_{f}$ of flavours one can reorganise the two-dimensional spinors in $N_{f} / 2$ four-dimensional spinors, for which a $U\left(N_{f}\right)$ chiral symmetry can be defined [25]. One can then meaningfully ask whether this symmetry is spontaneously broken down to $U\left(N_{f}\right) \rightarrow U\left(N_{f} / 2\right) \times U\left(N_{f} / 2\right)$ by the formation of a fermion-antifermion condensate, and, if so, if this happens at the onset of confinement. Given the doubling phenomenon, in this context one type of staggered fermions counts as two flavours.

Quite independently of this, one can also check whether localised modes appear at deconfinement. The change in dimensionality adds a twist to the issue, since two spatial dimensions 

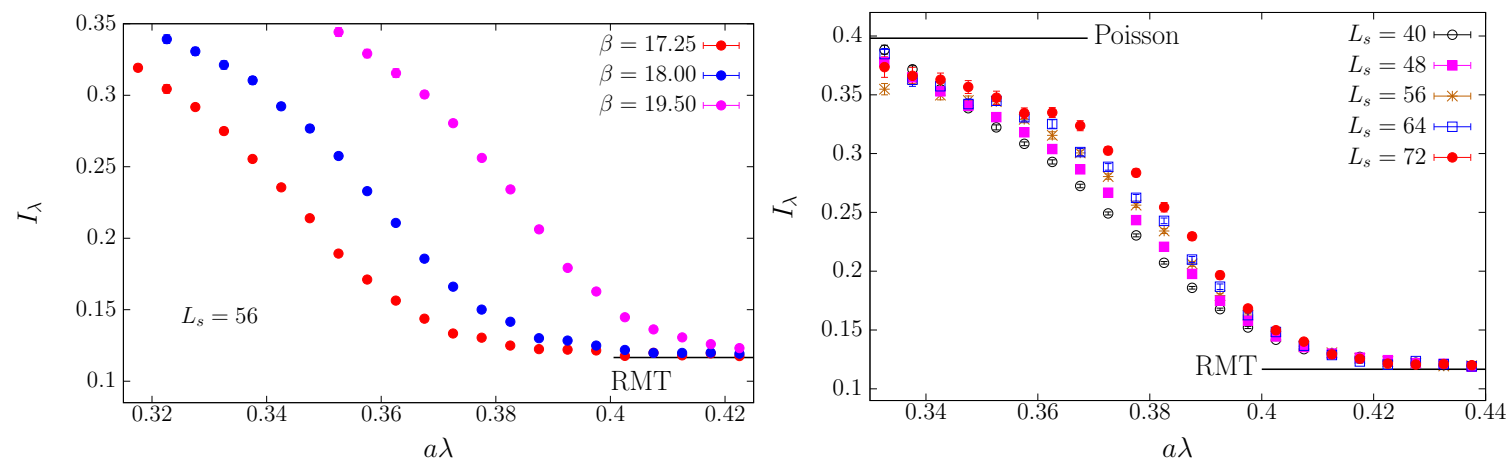

Figure 4: (Left.) Transition of $I_{\lambda}$ from the localised to the delocalised regime of the spectrum in the 2+1D $\mathrm{SU}(3)$ pure gauge theory for various $\beta$. (Right.) Same as above, but for fixed $\beta=19.5$ and various volumes.

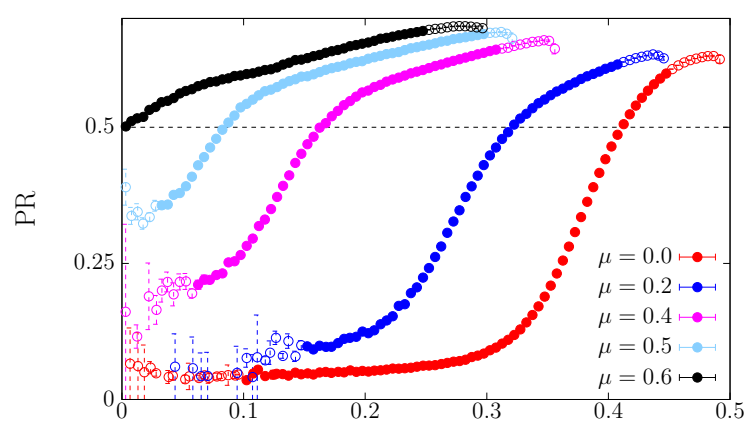

$a \lambda$

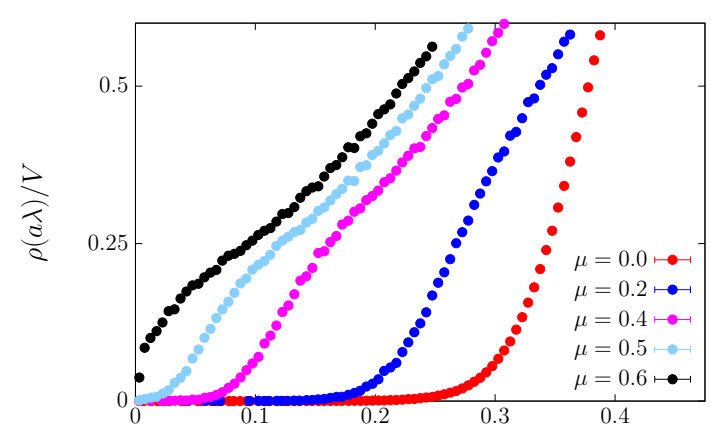

$a \lambda$

Figure 5: PR (left) and spectral density (right) in the 2+1D SU(3) pure gauge theory with an imaginary chemical potential.

are special for Anderson transitions, with the existence and the nature of the transition depending heavily on the details of the model.

In the remainder of this section I discuss some preliminary numerical results.

\section{1 $\mathrm{SU}(3)$ pure gauge theory}

As a first example I consider SU(3) pure gauge theory in 2+1D, studying the spectrum of the staggered Dirac operator. Deconfinement and chiral restoration have been shown long ago to take place at the same critical coupling $\beta_{c} \simeq 15$ [ [ deep in the confined phase, while localised modes are present at the low end of the spectrum in the deconfined phase, with a transition to delocalised modes at some critical point in the spectrum, which is qualitatively seen to decrease with temperature [see Fig. [4(left)].

An interesting aspect of the transition is that the curves of the spectral statistics for different volumes, instead of crossing at one point, seem to merge beyond some point in the spectrum [see Fig. 1 (right)]. This is typical of BKT-type Anderson transitions, and the same behaviour is observed in the analogous 2D AM [26]. Defining $\lambda_{c}$ as the merging point, one sees that it decreases with $\beta$. The expectation is that it will vanish at $\beta_{c}$, although this cannot be confirmed at this stage.

\subsection{SU(3) pure gauge theory with imaginary chemical potential}

If one changes the typical value of the Polyakov line phases or the temporal boundary con- 


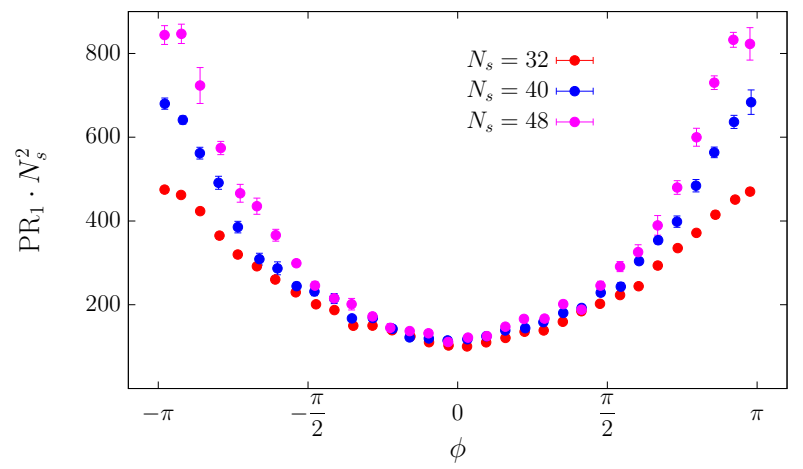

Figure 6: Average PR of the lowest eigenmode on U(1) gauge configurations with $\arg \bar{P}=\phi$ for various volumes (see text).

ditions, one changes the typical effective Matsubara frequencies, and thus the effective gap in the ordered phase. According to the "sea/islands" picture, this should affect the spectral density and the localisation properties of the low modes.

One way to test this is to study $\mathrm{SU}(3)$ pure gauge theory in 2+1D including an imaginary chemical potential $\mu$ in the Dirac operator, which in practice changes the temporal boundary conditions. According to the "sea/islands" picture, increasing $\mu$ should decrease the effective gap and thus push towards larger spectral density near the origin, and moreover should make localisation harder. In Fig. 1 (left) I show the PR throughout the spectrum: it is clear that it increases as $\mu$ is increased, so that the mobility edge is pushed down, until modes become delocalised. In Fig. 5 (right) I show the spectral density, which is indeed seen to increase with $\mu$.

\subsection{U(1) pure gauge theory}

Another interesting case is 2+1D U(1) pure gauge theory, which lacks another feature of QCD that should not matter for the connection between deconfinement, chiral restoration and localisation, namely the non-Abelian nature of the gauge group. Here in the deconfined phase the absolute value of the Polyakov loop develops a nonzero expectation value, while its phase fluctuates from configuration to configuration, and therefore so does the effective gap. Let $\bar{P}=(1 / V) \sum_{n} P(n)=$ $r e^{i \phi}$ be the spatially-averaged Polyakov loop on a given configuration. Studying the localisation properties of the lowest mode for different values of the phase $\phi$, one finds that it is localised for $\phi$ near zero, while it is delocalised for $\phi$ away from zero. This is also in agreement with the "sea/islands" picture.

\section{Conclusions and outlook}

Deconfinement, chiral symmetry restoration and localisation of the low modes of the Dirac operator are three closely connected phenomena, taking place at the same temperature in QCD and similar theories. The "sea/islands" picture and the Dirac-Anderson approach discussed in Section 3, together with numerical results in a quite diverse variety of models, support the idea that deconfinement is the driving force behind the other two phenomena.

Insights into these issues could come from the study of other QCD-related models, besides the $2+1$ dimensional ones discussed in Section 6. In particular, the effect of an imaginary chemical 
potential should be studied in the fully dynamical case in 3+1D. Moreover, the possible role of localisation should be studied in the SU(3) gauge theory with adjoint fermions, where deconfinement and chiral symmetry restoration take place at different temperatures. Finally, the possible role of topology should be investigated in detail.

\section{Acknowledgements}

This work was partly supported by grants OTKA-K-113034 and NKFIH-KKP126769.

\section{References}

[1] S. Borsányi et al. [Wuppertal-Budapest Collaboration], JHEP 1009 (2010) 073.

[2] A. Bazavov et al., Phys. Rev. D 93 (2016) 114502.

[3] G. Boyd, J. Engels, F. Karsch, E. Laermann, C. Legeland, M. Lütgemeier and B. Petersson, Nucl. Phys. B 469 (1996) 419.

[4] P. H. Damgaard, U. M. Heller, A. Krasnitz and T. Madsen, Phys. Lett. B 440 (1998) 129.

[5] P. de Forcrand and O. Philipsen, Nucl. Phys. B 673 (2003) 170.

[6] T. Banks and A. Casher, Nucl. Phys. B 169 (1980) 103.

[7] A. M. García-García and J. C. Osborn, Phys. Rev. D 75 (2007) 034503.

[8] T. G. Kovács, Phys. Rev. Lett. 104 (2010) 031601.

[9] T. G. Kovács and F. Pittler, Phys. Rev. Lett. 105 (2010) 192001.

[10] T. G. Kovács and F. Pittler, Phys. Rev. D 86 (2012) 114515.

[11] M. Giordano, T. G. Kovács and F. Pittler, Phys. Rev. Lett. 112 (2014) 102002.

[12] G. Cossu and S. Hashimoto, JHEP 1606 (2016) 056.

[13] L. Holicki, E. M. Ilgenfritz and L. von Smekal, arXiv:1810.01130 [hep-lat].

[14] P. W. Anderson, Phys. Rev. 109 (1958) 1492.

[15] B. I. Shklovskii, B. Shapiro, B. R. Sears, P. Lambrianides and H. B. Shore, Phys. Rev. B 47 (1993) 11487.

[16] L. Giusti and M. Lüscher, JHEP 0903 (2009) 013.

[17] K. Slevin and T. Ohtsuki, Phys. Rev. Lett. 82 (1999) 382.

[18] L. Ujfalusi, M. Giordano, F. Pittler, T. G. Kovács and I. Varga, Phys. Rev. D 92 (2015) 094513.

[19] F. Bruckmann, T. G. Kovács and S. Schierenberg, Phys. Rev. D 84 (2011) 034505.

[20] M. Giordano, T. G. Kovács and F. Pittler, JHEP 1504 (2015) 112.

[21] M. Giordano, T. G. Kovács and F. Pittler, JHEP 1606 (2016) 007.

[22] M. Giordano, T. G. Kovács and F. Pittler, Phys. Rev. D 95 (2017) 074503.

[23] M. Giordano, S. D. Katz, T. G. Kovács and F. Pittler, JHEP 1702 (2017) 055.

[24] T. G. Kovács and R. A. Vig, Phys. Rev. D 97 (2018) 014502.

[25] R. D. Pisarski, Phys. Rev. D 29 (1984) 2423.

[26] X. C. Xie, X. R. Wang and D. Z. Liu, Phys. Rev. Lett. 80 (1997) 3563. 